\title{
Spontaneous Renal Hemorrhage: Case Report and Review of the Literature
}

Subhash Paudel ( $\nabla$ subhash.900@gmail.com )

Beth Israel Deaconess Medical Center https://orcid.org/0000-0003-3825-8456

Peter Steinberg

Beth Israel Deaconess Medical Center

Bradley Denker

Beth Israel Deaconess Medical Center

\section{Research Article}

Keywords: Spontaneous renal hemorrhage, chronic kidney disease, conservative management

Posted Date: March 2nd, 2022

DOI: https://doi.org/10.21203/rs.3.rs-1139231/v1

License: (1) This work is licensed under a Creative Commons Attribution 4.0 International License.

Read Full License 


\section{Abstract}

Introduction:

Spontaneous renal hemorrhage (SRH) is an uncommon condition which is usually associated with renal neoplasms or vasculitis. Management of SRH is uniquely challenging in cases of solitary functioning kidney as the risks and benefits of surgical intervention and conservative management need to be carefully considered.

Case Presentation:

We report a case of a 67-year-old woman with recurrent bilateral nephrolithiasis with an atrophic right kidney who presented with left flank pain and acute kidney injury similar to prior episodes of nephrolithiasis. She was found to have SRH in her solitary functioning kidney and was managed non operatively.

Conclusion:

Clinical presentation of SRH can be similar to that of nephrolithiasis or pyelonephritis and a high index of suspicion is required to diagnose this life-threatening condition. Management of SRH is especially challenging in patients with solitary kidney. As this case illustrates, management decisions should be individualized and conservative care with close monitoring is an option in selective situations.

\section{Introduction:}

Spontaneous renal hemorrhage (SRH), also known as Wunderlich syndrome, is an uncommon condition characterized by non-traumatic subcapsular and perirenal hematoma. A meta-analysis of cases of SRH between 1985 and 1999 identified 165 cases $^{1}$ and a subsequent systematic review published in 2017 identified 102 more cases of SRH between 2000 and 2016. ${ }^{2}$ Renal neoplasms followed by vasculitis were identified as the most common etiology of SRH. ${ }^{1-3}$ Angiomyolipoma was the most the form of neoplasm and polyarteritis nodosa was the most common form of vasculitis associated with SRH. Flank pain, hematuria, anemia and shock were the most common presentation. ${ }^{2} \mathrm{CT}$ scan is the diagnostic modality of choice but has moderate sensitivity for detection of underlying etiology (sensitivity 0.57$)^{1}$. In the latest systematic review, the most common form of initial treatment was arterial embolization (42.2\%) followed by medical management $(29.4 \%)$ and only $27.5 \%$ of patients were initially treated surgically. ${ }^{2}$

\section{Case Presentation:}

We report an unusual case of spontaneous subcapsular and perinephric hematoma in a 67-year-old woman with solitary functioning kidney and long-standing chronic kidney disease due to obstructive uropathy. Her medical history included history of obesity, hypertension, hepatitis $\mathrm{C}$ and history of opioid abuse. Her surgical history was notable for laparoscopic gastric bypass surgery. 
Her renal function was normal prior to 6/2012 when she had symptomatic right sided obstructing kidney stone with hydronephrosis. This was associated with acute kidney injury (creatinine peaked at $1.5 \mathrm{mg} / \mathrm{dl}$ ) and required lithotripsy and ureteric stent placement. She had another episode of acute kidney injury 15 months later which was determined to be due to acute tubular necrosis (ATN) in the setting of a urinary tract infection and volume depletion and her creatinine peaked at $10.4 \mathrm{mg} / \mathrm{dl}$. She did not require renal replacement therapy and although her renal function improved, she had residual stage 4 chronic kidney disease (CKD) with creatinine around 2-2.6 mg/dl (estimated GFR $22 \mathrm{ml} / \mathrm{min}$ ). She was also incidentally found to have a right sided solid renal mass in 7/2013 on an MRI and was followed with serial imaging. This was suspected to be papillary renal cancer based on imaging and it remained stable on subsequent imaging studies.

In 1/2016, approximately 4 years after the initial episode of right sided hydronephrosis, she suffered from another episode of acute left sided flank pain. CT scan showed left sided obstructing stone in the ureter with associated hydronephrosis and atrophic kidney on the right side which measured around 8 centimeters. In the setting of obstruction of her presumably only functioning kidney, she developed severe $\mathrm{AKI}$ and her creatinine peaked at $13.2 \mathrm{mg} / \mathrm{dl}$. She required percutaneous nephrostomy tube placement on the left side followed by lithotripsy and ureteric stent placement. Following the resolution of hydronephrosis, her renal function improved and it remained stable over the next 4 years with creatinine between 2-2.6 mg/dl.

In 11/2020, she presented with sudden onset of left sided flank pain which was similar to her previous episodes of nephrolithiasis. She did not have hematuria or symptoms to suggest urinary tract infection. On examination, she was not in distress and vital signs on presentation to the emergency department were: temperature $98.1^{\circ} \mathrm{F}$, heart rate 82 beat per minute, blood pressure $92 / 51 \mathrm{mmHg}$, respiratory rate 16 per min and oxygen saturation $98 \%$ on room air. She was noted to have conjunctival pallor and left renal costovertebral angle tenderness. Evaluation (Table 1) was notable for acute anemia with hemoglobin of $6.3 \mathrm{~g} / \mathrm{dl}$ down from $10.2 \mathrm{~g} / \mathrm{dl}$ and acute renal injury with creatinine up to $5.3 \mathrm{mg} / \mathrm{dl}$. Urinalysis showed 210 RBCs and urine protein to creatinine ratio was $0.4 \mathrm{~g} / \mathrm{g}$.

CT of abdomen and pelvis without contrast showed left sided sub-capsular hematoma and surrounding peri-renal hematoma and contralateral atrophic kidney on the right side (Figure. 1). Renal ultrasound showed a complex collection on the left suggestive of peri-renal hematoma. Prior to this presentation, she had not sustained any trauma, undergone urological interventions, or kidney biopsy and she had not been taking any antiplatelet, non-steroidal analgesics or anticoagulants.

She was managed conservatively and received three units of packed red blood cell transfusion to keep hemoglobin above $7 \mathrm{~g} / \mathrm{dl}$. She developed hyperkalemia (potassium $6.3 \mathrm{meq} / \mathrm{L}$ ) and acidemia due to metabolic acidosis (venous PH 7.22 with bicarbonate of 15). She was given isotonic sodium bicarbonate (150 meq of sodium bicarbonate in $1 \mathrm{~L}$ of $\mathrm{D} 5 \mathrm{~W}$ ) along with intravenous furosemide with improvement in acidemia and hyperkalemia. She did not need renal replacement therapy and her renal function improved but did not recover back to her previous level of functioning. At 8 months after discharge, her creatinine 
was $3.7 \mathrm{mg} / \mathrm{dl}$ (eGFR $13 \mathrm{ml} / \mathrm{min}$ ) and ultrasound of her kidneys showed near complete resolution of the hematoma. (Figure 2)

\section{Discussion And Conclusions:}

Spontaneous renal hemorrhage is an unusual condition that is usually associated with an underlying renal neoplasm or vasculitis. We present a case of SRH in a woman with prior history of kidney stones and an atrophic kidney who developed SRH in her solitary functioning kidney. She presented with signs and symptoms that were similar to her previous episodes of kidney stones. Although she had a suspicious mass in her contralateral atrophic kidney, prior imaging had not shown any evidence of neoplasm in the affected kidney. Therefore, the hemorrhage on this side is unlikely to be explained by an underlying renal cancer.

Due to her advanced chronic kidney disease, further diagnostic evaluation of the etiology was deferred as it was thought to be unlikely to change her management. Although she had history of hepatitis $\mathrm{C}$, it had been in remission after treatment and thus polyarteritis nodosa was considered to be very unlikely. Uremic platelet dysfunction was considered to be a possible risk factor for her SRH.

Given her stage $V$ chronic kidney disease with eGFR of $\sim 13 \mathrm{ml} / \mathrm{min}$, a non-operative treatment approach with watchful monitoring of her renal function and serial imaging was done. Based on recent systematic review, minimally invasive and non-operative management have become more common management approaches for SRH over the past several years. ${ }^{2}$ In conclusion, spontaneous renal hemorrhage can mimic acute nephrolithiasis and can rarely occur in the absence underlying lesion or vasculitis.

\section{List Of Abbreviations:}

ATN: acute tubular necrosis, CKD: chronic kidney disease, eGFR: estimated glomerular filtration rate, SRH: spontaneous renal hematoma

\section{Declarations:}

Ethics approval and consent to participate: Approval by ethics committee not applicable for case report Consent for publication: Written consent for publication has been obtained from the patient

Availability of data and Materials: The datasets used and/or analysed during the current study are available from the corresponding author on reasonable request.

Competing interests: The authors declare that they have no competing interests.

Funding: None 
Authors' contributions: SP, BD and PS were directly involved in taking care of the patient. SP prepared the initial draft of the manuscript, BD reviewed the draft of the manuscript with additions. PS selected the relevant images for submission. All authors read and approved the final manuscript.

Acknowledgements: Not applicable

\section{References:}

1. Zhang JQ, Fielding JR, Zou KH. Etiology of spontaneous perirenal hemorrhage: A meta-analysis. $J$ Urol. 2002;167(4 I):1593-1596. doi:10.1016/s0022-5347(05)65160-9

2. Ahn T, Roberts MJ, Navaratnam A, Chung E, Wood S. Changing etiology and management patterns for spontaneous renal hemorrhage: a systematic review of contemporary series. Int Urol Nephrol. 2017;49(11):1897-1905. doi:10.1007/s11255-017-1694-8

3. Kim JW, Kim JY, Ahn ST, et al. Spontaneous perirenal hemorrhage (Wunderlich syndrome): An analysis of 28 cases. Am J Emerg Med. 2019;37(1):45-47. doi:10.1016/j.ajem.2018.04.045

\section{Table:}


Table 1

Laboratory data

\begin{tabular}{|llll|}
\hline & $2-3$ months prior to presentation & At presentation & At discharge \\
\hline Hemoglobin $(\mathrm{g} / \mathrm{L})$ & 102 & 63 & 82 \\
\hline Hematocrit $(\%)$ & 31.0 & 18.6 & 24.9 \\
\hline Leukocyte Count $\left(10^{9} / \mathrm{L}\right)$ & 5.5 & 10.1 & 6.9 \\
\hline Platelet Count $\left(10^{9} / \mathrm{L}\right)$ & 135 & 142 & 165 \\
\hline $\mathrm{PT}(\mathrm{seconds}) / \mathrm{INR}$ & & $12.4 / 1.1$ & $12.4 / 1.1$ \\
\hline Sodium (mmol/L) & 141 & 128 & 136 \\
\hline Potassium (mmol/L) & 5.0 & 6.3 & 4.0 \\
\hline Chloride (mmol/L) & 107 & 100 & 98 \\
\hline Bicarbonate (mmol/L) & 22 & 15 & 22 \\
\hline Blood Urea Nitrogen (mmol/L) & 12.85 & 24.99 & 26.06 \\
\hline Creatinine (micromol/L) & 194.48 & 468.52 & 362.44 \\
\hline Calcium, total (mmol/L) & 2.33 & 2.25 & 2.23 \\
\hline Phosphorus (mmol/L) & 1.68 & 1.81 & 1.84 \\
\hline Magnesium (mmol/L) & 0.78 & 0.82 & 0.70 \\
\hline
\end{tabular}

Figures 


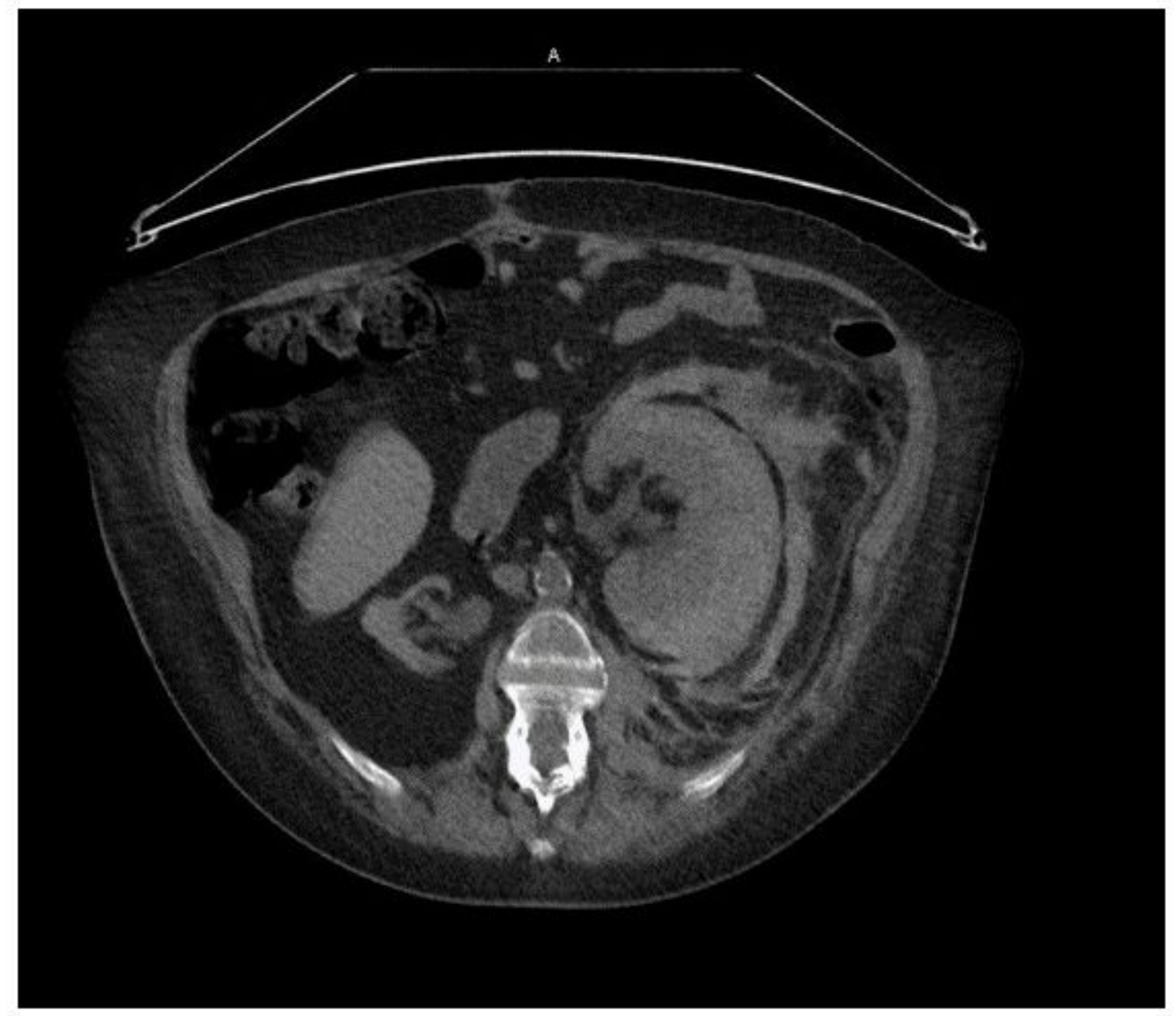

Figure 1

CT without contrast showing left preinephric hematoma 


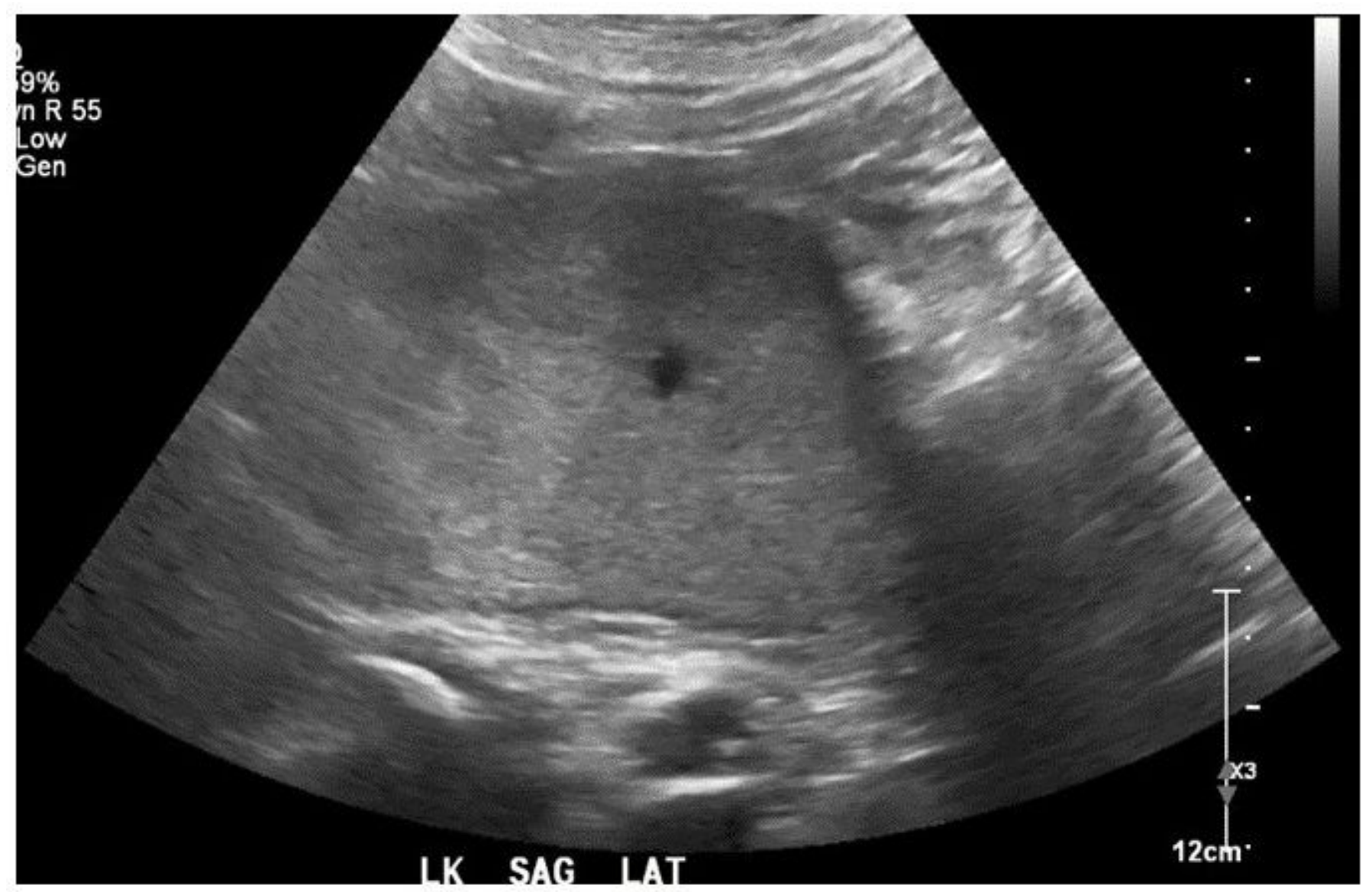

Figure 2

Ultrasound at 6 months showing near complete resolution of hematoma

\section{Supplementary Files}

This is a list of supplementary files associated with this preprint. Click to download.

- CAREchecklistEnglish2013.pdf 